\title{
The band and slough technique is effective for management of diminutive type 1 gastric and duodenal neuroendocrine tumors
}

\section{다 (1) $(2)=$}

\author{
Authors \\ Fadi Hawa ${ }^{1}$, Zeyad Sako², Than Nguyen ${ }^{4}$, Andrew T. Catanzaro ${ }^{3}$, Eugene Zolotarevsky ${ }^{5}$, Angela N. Bartley ${ }^{6}$, Naresh T.
} Gunaratnam ${ }^{3}$

Institutions

1 Department of Internal Medicine, St. Joseph Mercy Ann Arbor Hospital, Ypsilanti, Michigan, United States

2 Department of Hematology and Oncology, Ascension St. John Hospital, Detroit, Michigan, United States

3 Department of Gastroenterology and Hepatology, Huron Gastroenterology Associates, St. Joseph Mercy Ann Arbor Hospital, Ypsilanti, Michigan, United States

4 Department of Gastroenterology and Hepatology, Kaiser Permanente Vacaville Medical Center, Vacaville, California, United States

5 Department of Gastroenterology and Hepatology, Spectrum Health Hospitals, Grand Rapids, Michigan, United States

6 Department of Pathology, St. Joseph Mercy Ann Arbor Hospital, Ypsilanti, Michigan, United States

submitted 3.11 .2019

accepted after revision $\quad 3.2 .2020$

Bibliography

DOI https://doi.org/10.1055/a-1119-6698 |

Endoscopy International Open 2020; 08: E717-E721

(c) Georg Thieme Verlag KG Stuttgart · New York elSSN 2196-9736
Corresponding author

Naresh T. Gunaratnam, MD, Huron Gastroenterology

Associates, 5300 Elliott Drive, Ypsilanti, MI 48197

Phone: +1-734-714-0455

gunaratnamn@hurongastro.com

\section{ABSTRACT}

Background and study aims Endoscopic resection is recommended as initial treatment for early-stage gastric and duodenal neuroendocrine tumors (G-NETs and D-NETs). However, it can cause serious adverse events. We aimed to evaluate the efficacy and safety of the band and slough (BAS) technique as a novel and less aggressive endoscopic therapy for management of such tumors.

Four patients, three diagnosed with $<10-\mathrm{mm}$ D-NET and one with 10-mm type I G-NET, were treated with the BAS technique without endoscopic resection. Initial follow-up endoscopy at 3 months was done to assess for residual tumor. Subsequent endoscopic surveillance was performed. After one session of banding, all patients achieved complete remission at 3-month follow-up. No tumor recurrence was detected on repeat biopsy at 12-month surveillance endoscopy. None of the patients developed any adverse events including bleeding or perforation.

The BAS technique may prove to be a safe and effective endoscopic therapy for diminutive, non-metastatic type 1 G-NETs and D-NETs. Studies of larger scale and longer follow-up periods are needed to corroborate these findings.

\section{Introduction}

Gastrointestinal neuroendocrine tumors (GI-NETs) are rare tumors. Gastric NETs (G-NETs) and duodenal NETs (D-NETs) are considered the most common upper GI-NETs [1]. Diagnosis of these lesions is currently on the rise, due to widespread utilization of upper endoscopy and improvements in classification of these tumors.
Regardless of the type (G-NETs or D-NETs), endoscopic resection is recommended as an initial treatment for tumors that are not yet at advanced stages [2,3]. Surgery is recommended for all advanced cases either as an initial therapy or following endoscopic resection [3,4].

GI-NETs eligible for endoscopic management are those of small tumor size (Type I G-NETs $\leq 10 \mathrm{~mm}$ and D-NETs $\leq 10 \mathrm{~mm}$ ) as they are less likely to be associated with muscularis propria invasion or metastasis [3-6]. Current endoscopic techniques, 
both endoscopic mucosal resection (EMR) and endoscopic submucosal dissection (ESD), are alternatives to surgical management but are associated with risk of adverse events (AEs) including bleeding, perforation and stricture formation secondary to electrocautery use [7]. These risks are even higher with tumors of the duodenal bulb due to the thin duodenal wall and its increased vasculature.

The band and slough (BAS) technique, also known as endoscopic band ligation without resection (BWR), has been used for the management of mucosal lesions in the gastrointestinal tract with varying degrees of success ranging from $75 \%$ to $100 \%$ [8]. We have previously reported outcomes of the BAS technique in management of non-ampullary duodenal adenomas without the use of electrocautery [9]. The BAS technique works by compressive tissue ischemia resulting in adenoma sloughing off without the use of electrocautery. Thus, reducing risk of AEs associated with EMR and ESD. This technique has not been thoroughly evaluated for management of gastric and duodenal neuroendocrine tumors. Accordingly, we examined outcomes of the BAS technique as a novel and less aggressive endoscopic method to effectively manage diminutive $(\leq 10 \mathrm{~mm})$ gastric and duodenal neuroendocrine tumors.

\section{Patients and methods}

\section{Patients}

The study was approved by the St. Joseph Mercy Ann Arbor Hospital Institutional Review Board. All eligible patients were adults of age 18 years or older with biopsy proven, low grade (G1), and non-metastatic Type $1 \mathrm{G}-\mathrm{NETs} \leq 10 \mathrm{~mm}$ and D-NETs $\leq 10 \mathrm{~mm}$. Patients with metastatic disease evident on multislice computed tomography scan (regional lymph node and/or distant metastasis), poor histological differentiation, high grade (G2-G3), G-NETs $>10 \mathrm{~mm}$, type II and III G-NETs or D-NETs $>10 \mathrm{~mm}$ were excluded from the study. Before the endoscopic procedure, diagnosis of NETs was confirmed by a biopsy consistent with a low grade (G1), well-differentiated NET. As per ENETS consensus guidelines for management of gastroduodenal neuroendocrine tumors, endoscopic ultrasonography (EUS) was conducted for type $1 \mathrm{G}$-NETs to assess the depth of invasion and regional metastasis [4]. However, EUS was not performed in the included patients with D-NETs given the small tumor size $\leq 10 \mathrm{~mm}$ [3].

\section{Methods}

Risks and benefits of the BAS technique versus EMR or ESD were discussed with the patients prior to the procedure and an informed consent was obtained. All procedures were performed by a single endoscopist on an outpatient basis, with the patient under conscious sedation. The neuroendocrine tumors were assessed for absence of alarming features (eg, small size, nonulcerated, absence of central depression). Polyp size was estimated based on visual assessment ( $\triangleright$ Fig. 1).

The Speedband Superview Super (Boston Scientfic, Natick, Massachusetts, United States) banding kit was fitted onto a gastroscope in a standard fashion. The tumor was suctioned into the cap, and rubber bands were deployed at its base. Up to four bands were placed at the discretion of the endoscopist.

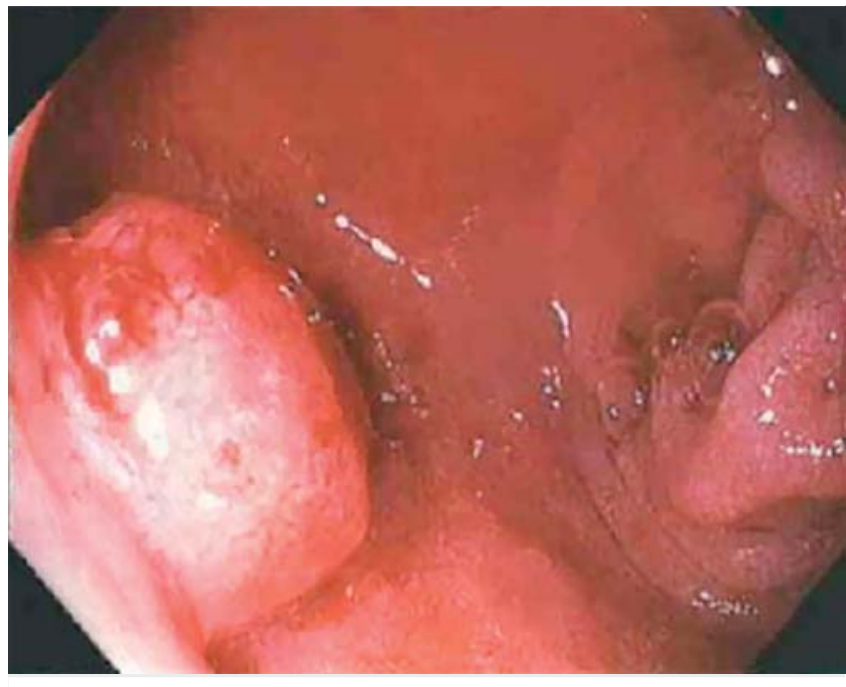

- Fig. 1 Endoscopic view of gastrointestinal neuroendocrine tumor in the duodenal bulb.

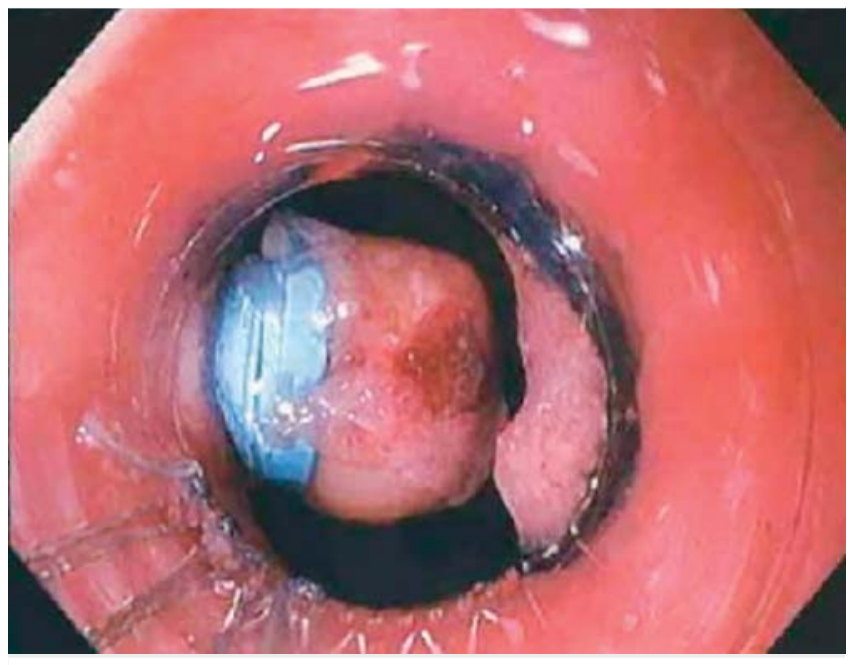

- Fig. 2 Pseudopolyp formation after band placement.

When more than one band was placed at a site, the second band was deployed deeper to the first band. This resulted in forming a prominent pseudopolyp ( $>$ Fig. 2). Endoscopic resection was not performed and the polyp was allowed to slough off spontaneously. Location of the GI-NET after banding was marked with submucosal India ink injection to facilitate surveillance for residual disease and tumor recurrence.

Patients were observed for 60 minutes for acute post-procedure adverse events. They were subsequently discharged home when no immediate adverse events were noted. Patients were also contacted via a telephone call by an endoscopy nurse after 24 hours to identify any adverse events that were not noted immediately after the procedure.

Follow-up endoscopic assessment was performed at 3 months for all patients with a repeat upper gastrointestinal endoscopy ( $>$ Fig.3). During which, the treatment site was identified by the tattoo and examined carefully by the endos- 


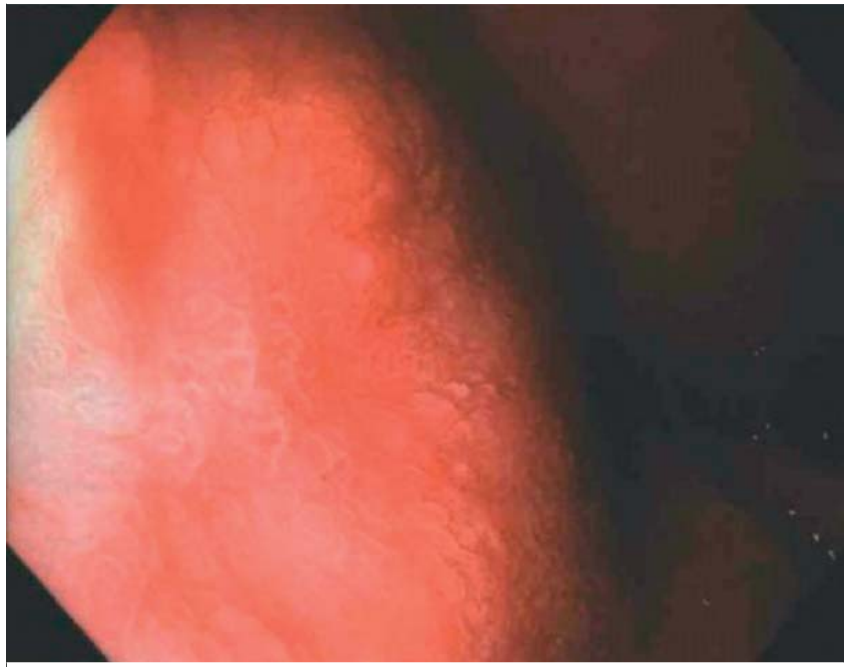

Fig. 3 Complete eradication of the neuroendocrine tumor at 3 months after a single banding session.

copist followed by extensive biopsies to assess for any residual disease. Surveillance endoscopy was then scheduled at a 12month follow-up interval.

The primary end point was complete histopathologic remission of GI-NETs confirmed by an expert gastrointestinal pathologist at 3-month follow-up ( $\triangleright$ Fig. 4).

\section{Results}

\section{Patients and GI-NET characteristics}

Four patients with an established diagnosis of GI-NET referred for therapy between 2015 and 2018 met the study inclusion criteria and subsequently underwent the BAS technique. All patients had a prior upper endoscopy conducted for evaluation of dysphagia, abdominal pain, or diarrhea. Initial endoscopy revealed incidental findings of polypoidal lesions in the gastric fundus or the duodenal bulb with biopsies consistent with NETs. One patient was diagnosed with a $10-\mathrm{mm}, \mathrm{G1}$, well-differentiated, type $1 \mathrm{G}-\mathrm{NET}$. EUS revealed submucosal extension without invasion into the muscularis propria. The tumor was uniquely located within the gastric fundus, $2 \mathrm{~cm}$ beyond the gastroesophageal junction which was technically challenging for EMR or ESD to be performed. The remaining three patients were diagnosed with $<10 \mathrm{~mm}, \mathrm{G1}$, well-differentiated and nonfunctioning, D-NETs located in the duodenal bulb. EUS was not performed given the small size of the D-NETs and the low histological grade. However, none of the patients had evidence of local or distant metastasis on multislice CT scan.

Patients and GI-NET characteristics, specifics of the procedures and outcomes are summarized in $>$ Table 1.

\section{Treatment outcomes}

Complete remission of the GI-NET was achieved in $100 \%$ of patients at 3 months follow-up, with only one session of banding. All patients have completed their 12-month follow-up without evidence of tumor recurrence on repeat upper endoscopy.

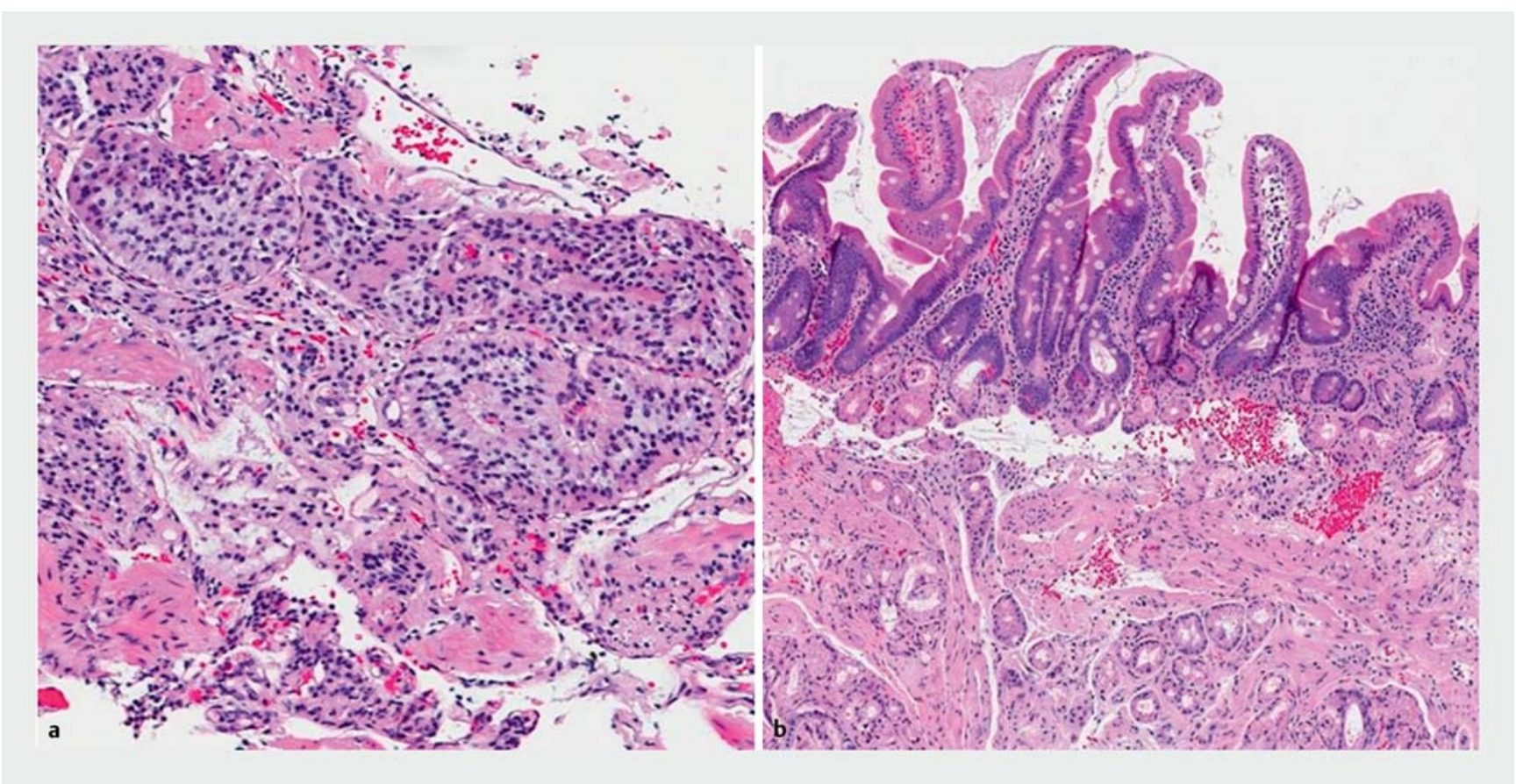

Fig. 4 a Well-differentiated neuroendocrine tumor in duodenal submucosa, $10 \times$ magnification. b Non-neoplastic duodenal mucosa with benign submucosal glands on biopsy obtained at 3-month surveillance, $5 \times$ magnification. 
- Table 1 Patient and GI-NET characteristics and specifics of the procedure.

\begin{tabular}{|l|c|}
\hline Number of patients & \multicolumn{1}{|c|}{4} \\
\hline Male, $\%$ & 50 \\
\hline Patient age, average (range), years & $70(66-77)$ \\
\hline GI-NETs Size, mean (range), mm & $8(6-10)$ \\
\hline Location of GI-NET, no. of patients & \\
\hline - Gastric Fundus & 1 \\
\hline - Duodenal Bulb & 3 \\
\hline Findings, no. of patients & \\
\hline - Type I G-NET & 1 \\
\hline - D-NET & 3 \\
\hline No. of banding sessions per patient & 1 \\
\hline No. of bands deployed per session, mean & 2.75 \\
\hline No. of patients at each follow-up interval & \\
\hline - 3 months & 4 \\
\hline - 12 months & 4 \\
\hline - 24 months & 1 \\
\hline $\begin{array}{l}\text { GI-NET gastric neuroendocrine tumor; D-NET, duodenal neuroendocrine } \\
\text { tumor }\end{array}$ \\
\hline
\end{tabular}

\section{Adverse events}

There was no bleeding, pain, perforation, or stricture formation after any intervention.

\section{Discussion}

This study demonstrates that the BAS technique is a safe and effective endoscopic treatment for diminutive ( $\leq 10 \mathrm{~mm})$, type 1 G-NETs and D-NETs. This is evident by the complete eradication of the GI-NET at 3-month follow-up after one session of banding only.

The BAS technique works by compressive tissue ischemia resulting in sloughing off and eradication of the tumor. Applying one band only is usually sufficient to induce the compressive tissue ischemia and is associated with lower risks of gastroduodenal wall tear and bleeding. In addition, endoscopic band ligation can effectively remove submucosal tumors and can also capture tumors originating from the muscularis propria without the use of electrocautery [10]. Therefore, it is expected to be associated with lower risks of bleeding, transmural injury and perforation which are known adverse events to EMR or ESD [7]. These risks are more pronounced with duodenal lesions as compared to gastric lesions which can be attributed to the thin duodenal wall and its increased vascularity. However, the BAS technique can also offer a safer endoscopic approach for gastric lesions that are technically challenging to undergo EMR or ESD due to difficult locations with increased risk of procedure-related AEs. Furthermore, the BAS technique offers more feasibility when compared to EMR or ESD as it does not require advanced endoscopy training and can be performed by a general gastroenterologist. Fortunately, all patients were able to be discharged home directly after the procedure with no need for hospitalization. No immediate, early or delayed postprocedure adverse events were reported for any of the patients.

The main limitations of the study are the small sample size given the rare nature of GI-NETs, need for longer follow-up periods, and lack of direct comparison to endoscopic resection techniques. Therefore, it is difficult to prove superiority or non-inferiority of the BAS technique. Further limitations include inability to assess the deep margins of the resection and lack of tissue retrieval at the time of therapy. This raises the concern for increased risk of residual disease and tumor recurrence. However, the diagnoses of GI-NETs were based on previously obtained biopsies, confirmed by an expert gastrointestinal pathologist. In addition, the tumors were carefully examined by the endoscopist for any signs of tumor invasion or malignant potential. Furthermore, close endoscopic follow-up was ensured to minimize the risk of missing a residual or recurrent tumor. In our patients, no residual or recurrent tumor was noted in any of the scheduled follow-up visits.

The success of the BAS technique is further demonstrated by the histological remission of GI-NETs with up to 12-month follow-up in D-NET patients and up to 24-month follow-up in our type 1 G-NET patient. Patients are currently scheduled for their follow-up endoscopic examination according to ENETS guidelines. Thus far, no acute or delayed AEs related to the procedure were observed in our patients.

\section{Conclusion}

In conclusion, the band and slough endoscopic technique may prove to be a safe, less aggressive, and effective alternative to endoscopic mucosal resection and endoscopic submucosal dissection in management of diminutive $(\leq 10 \mathrm{~mm})$, low-grade (G1), well-differentiated, type 1 gastric neuroendocrine tumors and duodenal neuroendocrine tumors. Studies of larger scale and longer follow-up periods are needed to corroborate these findings.

\section{Competing interests}

Dr. Gunaratnam is a speaker for Nestle Health Science and the Cofounder and Chief Medical Officer for Lean Medial LLC, an endoscopic weight loss device company.

\section{References}

[1] Sato Y, Hashimoto S, Mizuno K et al. Management of gastric and duodenal neuroendocrine tumors. World J Gastroenterol 2016; 22: 6817

[2] Li TT, Qiu F, Qian ZR et al. Classification, clinicopathologic features and treatment of gastric neuroendocrine tumors. World J Gastroenterol 2014; 20: 118-1125 
[3] Delle Fave G, Kwekkeboom D], Van Custem E et al. ENETS consensus guidelines for the management of patients with gastroduodenal neoplasms. Neuroendocrinology 2012; 95: 74-87

[4] Delle Fave G, O'Toole D, Sundin A et al. ENETS consensus guidelines update for gastroduodenal neuroendocrine neoplasms. Neuroendocr 2016; 103: 119-124

[5] Kulke MH, Shah MH, Benson AB et al. Neuroendocrine tumors, version 1. 2015. J Nat Comp Cancer Net 2015; 13: 78-108

[6] Scherübl H, Cadiot G, Jensen RT et al. Neuroendocrine tumors of the stomach (gastric carcinoids) are on the rise: small tumors, small problems? Endoscopy 2010; 42: 664-671
[7] Raju GS. Complications of endoscopic mucosal resection and endoscopic submucosal dissection. Tech Gastrointest Endosc 2011; 13: 91-94

[8] Khara HS, Sholvn G], Johal AS et al. Endoscopic banding without resection (BWR) technique for treatment of diminutive neuroendocrine tumors in the duodenum. Endosc Int Open 2019; 7: E302-E307

[9] Koritala T, Zolotarevsky E, Bartley AN et al. Efficacy and safety of the band and slough technique for endoscopic therapy of nonampullary duodenal adenomas: a case series. Gastrointest Endosc 2015; 81: 985-988

[10] Xia FZ, Shen Z, Chen HT et al. Endoscopic banding ligation can effectively remove the submucosal tumor. Int J Clin Exper Med 2015; 8: 1494 